\title{
Digitale Anwendungen im Praxisalltag: Yes we (s)can oder wie weit wollen wir gehen?
}

\section{Digital Applications in Everyday Practice: Yes we (s)can or how far do we want to go?}

\author{
Autor \\ Simon Graf \\ Institut \\ Privatpraxis Belp, Schweiz \\ Schlüsselwörter \\ digitale Kieferorthopädie, CAD/CAM-Retainer, metallge- \\ druckte Apparaturen, Rapid-Prototyping \\ Key words \\ digital orthodontics, CAD/CAM retainers, metal-printed \\ appliances, rapid prototyping \\ Bibliografie \\ DOI https://doi.org/10.1055/s-0043-116702 \\ Inf Orthod Kieferorthop 2017; 49: 171-176 \\ (c) Georg Thieme Verlag KG Stuttgart · New York \\ ISSN 0020-0336 \\ Korrespondenzadresse \\ Dr. med. dent. Simon Graf \\ Praxis für Kieferorthopädie \\ Eichenweg 23 \\ 3123 Belp \\ Schweiz \\ simon.graf@unibas.ch
}

\section{ZUSAMMENFASSUNG}

Die Digitalisierung ist ein Trend, der zunehmend auch den Bereich der klinischen Kieferorthopädie erobert. Die unglaublich raschen Entwicklungen auf dem Gebiet der technischen Herstellungsverfahren bedeuten zugleich auch Zeitersparnis und eine geringere Belastung für den Patienten. Selten war es mit so wenig Aufwand möglich, so viele unterschiedliche Aspekte einer einfachen kieferorthopädischen Apparatur mit nur einem Tastenklick zu verändern und individuell anzupassen. Wie sieht also die Praxis der Zukunft aus? Der vorliegende Artikel gibt konkrete Einblicke in die wunderbare Welt der digitalen Praxis und beleuchtet aus der Sicht eines Kieferorthopäden die verschiedenen Aspekte der digitalen Einflüsse und gibt Beispiele für die digitale Umsetzung.

\section{ABSTRACT}

Digitisation is a trend which is also increasingly taking over the area of clinical orthodontics. The incredibly rapid developments in the field of technical production processes mean that time is saved at the same time and there is less of a burden for the patient. Rarely has it been possible with so little effort to change and individually adjust so many different aspects of a simple orthodontic appliance with just a single click of a button. So what does the practice of the future look like? This article gives specific insights into the wonderful world of digital practice and, from the perspective of an orthodontist examines the various aspects of digital influences and gives examples of digital implementation.

\section{Einleitung}

Die zunehmende Digitalisierung ist aus unserem Alltag kaum mehr wegzudenken. Wir begegnen ihr fortwährend. Wann fährt der nächste Bus? Ein kurzer Blick auf das Smartphone und wir sind informiert. Die nächsten Ferien? Fünf Minuten „googeln“, Angebote vergleichen und schon ist der nächste Urlaub gebucht. Keine lästigen Umwege mehr und keine endlosen Wartezeiten in einem Reisebüro.

Der Trend der zunehmenden Digitalisierung lässt sich auch für die Zahnmedizin [1] und den Bereich der klinischen Kieferorthopädie [2-6] deutlich erkennen. Bringt die zunehmende Digitalisie- rung also wirklich Vorteile oder konkret gefragt, erleichtert sie unseren täglichen kieferorthopädischen Alltag? Was bringt uns wirklich weiter? Was wirft uns zurück? Und, wie können wir die digitalen Applikationen sinnvoll nutzen, um die Behandlung für unsere Patienten effizienter und besser zu gestalten?

Es folgen Anwendungs- und Diagnosemöglichkeiten, strukturelle und organisatorische Voraussetzungen, Grenzen und mögliche Stolpersteine digitaler Anwendungen sowie konkrete Anwendungsbeispiele aus der Sicht des Autors, eines seit 2014 überzeugten Anwenders der digitalen Kieferorthopädie. 


\section{Anwendungs- und Diagnosemöglichkeiten}

Bei der digitalen Praxisorganisation hat der Computer die Krankengeschichte auf Papier schon länger abgelöst. Ein papierloses und damit karteikartenfreies Arbeiten ermöglicht grundsätzlich eine ausgesprochen effiziente und straffe Praxisorganisation - alle Unterlagen sind jederzeit von allen Computerarbeitsplätzen der Praxis abrufbar. Das ist ein klarer Vorteil für das Praxispersonal, da keine wertvolle Zeit für das Zusammenstellen von Patientenunterlagen verloren geht. Praxisabläufe, Patientenstruktur, Behandlungsplanung und Terminbuch sind jederzeit transparent darstellbar und sorgen für ein übersichtliches Praxismanagement. Dazu kommt die rasch, meistens bereits selbstständig im Hintergrund ablaufende Diagnostik, wie z. B. die Modell- und Platzanalyse nach Tonn und Bolton der kieferorthopädischen Software. Natürlich können hier die unterschiedlichsten Analysen abgerufen werden.

Die Digitalisierung erleichtert auch das Abrechnungswesen, ermöglicht konkret eine prozessorientierte Abrechnung in bereits vorkonfigurierten Abrechnungskomplexen, ein integriertes Qualitätsmanagement sowie ein transparentes Controlling. Dies ist nicht nur für die Bürokraft in der Praxis eine Erleichterung, sondern auch für den Behandler, der die Einträge und Tarifposten direkt in die digitale Karteikarte eingeben und erfassen kann. Das führt zu geringeren finanziellen Verlusten, da die direkte digitale Dokumentation am Behandlungsplatz weniger zum Vergessen von Behandlungsschritten und Eintragungen führt.

Durch gezielte Vorinformationen nach dem ersten Patient-ArztKontakt via E-Mail können mögliche Unklarheiten im Vorfeld eines ersten Termins geklärt werden. Terminkoordinationen lassen sich schnell durch eine Praxismanagementsoftware erledigen, ohne dass die Bürokraft durch Telefonate gebunden ist. Eine Behandlung betreffende Fragen können durch das Bereitstellen von Anschauungsmaterial entweder auf einer Homepage oder via E-Mail einfach ohne zusätzliche Einbindung von Mitarbeitern beantwortet werden.

Das Patientenmanagement kann aber auch beliebig bis zur selbstständigen Online-Terminvergabe für die Patienten ausgebaut werden. So empfängt z. B. das Programm IEE-Systems (Michael Visse) den Patienten nach individueller und passwortgeschützter Anmeldung direkt mit Willkommens-E-Mails, versorgt ihn mit relevanten Informationen und bietet ihm sogar die Möglichkeit persönliche Patientenunterlagen auf einer Smartphone-App abzurufen und damit den Behandlungsfortschritt selbst zu verfolgen.

Ein weiteres wichtiges Einsatzgebiet ist die digitale Fotografie, die durch immer höhere Auflösungen und standardisierte Aufnahmen bis hin zur Stereofotografie das Erfassen von Abweichungen zwischen intraoralen oder extraoralen Befunden ermöglicht. Digitale Bilder helfen Behandlungen zu planen, welche über die Klasse I im Munde hinausgehen und darauf abzielen, ein möglichst harmonisches Lachen zu kreieren (Digital Smile Design). Sie helfen Behandlungsabläufe und -fortschritte zu dokumentieren oder Aufklärungen zu Behandlungsverfahren und -konzepten zu unterstützen.

Die 2-dimensionale digitale Röntgentechnologie ermöglicht eine digitale Erfassung von Befunden und erleichtert die Diagnostik (z. B. digitale FRS-Auswertung). Speziell die Auswertung und Überlagerung von Fernröntgenseitenbildern ist mit heutigen kieferorthopädischen Analyseprogrammen (z. B. OnyxCeph, Chiem- see, Deutschland) sehr vereinfacht und effizienter. Digitale Röntgenbilder können direkt am Computer ausgewertet und Helligkeit und Kontrast einfach justiert werden. Auf diese Weise helfen sie dem Kieferorthopäden zu einer schnellen und genauen Diagnose zu gelangen. Ein weiterer wichtiger Vorteil ist die gegenüber der klassischen Analogtechnik um bis zu 90 \% reduzierte Strahlenbelastung. Dies liegt vor allem daran, dass die digitale Speicherfolie gegenüber dem konventionellen Röntgenfilm wesentlich empfindlicher ist. Digitale Röntgenbilder weisen zudem eine höhere Bildqualität auf und bilden Zahn- und Knochenstrukturen hervorragend ab. Zudem können digitale Röntgenbilder einfach an Patienten, Kollegen und Mitbehandler übermittelt werden. Damit gehören qualitativ schlechte Kopien der Vergangenheit an. Zudem schont die Digitaltechnik auch die Umwelt: Analoge Röntgenfilme und vor allem die entsprechenden Entwicklungschemikalien, wie sie beim konventionellen Röntgen notwendig waren, entfallen vollständig. Auch die 3-dimensionale digitale Volumentomographie (DVT) [7-9] ist in unserer Diagnostik etabliert, insbesondere, wenn es z. B. um verlagerte Zähne geht.

Ein weiterer Bereich ist die digitale Abformung. 1985 führte Sirona (Siemens, Bensheim, Deutschland) lichtoptische, digitale Zahnabformungen in der rekonstruktiven Zahnmedizin ein. Beginnend von Einzelzahn-Scans führte die Entwicklung rasch weiter zu Scans von Quadranten und kompletten Zahnbögen (z. B. 3Shape, Kopenhagen, Dänemark).

Die immer präziseren lichtoptischen Abformungen machen herkömmliche Alginatabformungen obsolet, insbesondere was das Handling und die Präzision betrifft. Wissenschaftliche Untersuchungen zeigen mittlerweile eine ausreichend hohe Genauigkeit für die digitale Abformung [10-14]. Obwohl bspw. ein Quadranten-Scan grundsätzlich genauer ist als ein Ganzkieferscan, ist die Scan-Qualität zur Herstellung von kieferorthopädischen Apparaturen ausreichend genau. In diesem Zusammenhang zeigen verschiedene Intraoralscanner gute Ergebnisse [14]. Der Anwender muss bei einem korrekten Scanablauf keine Passungsschwierigkeiten befürchten.

\section{Strukturelle und organisatorische Voraus- setzungen}

Wie muss die moderne digitale Praxis technisch ausgestattet sein? Natürlich ist eine gewisse digitale Grundausrüstung erforderlich.

In jedem Fall ist eine adäquate Vernetzung mit Netzwerkkabeln (LAN) oder einem leistungsfähigen kabellosen Netzwerk (WLAN) vonnöten und dies in Kombination mit einem Server mit einer Leistung, die den heutigen Möglichkeiten entspricht. Wegen des rasend schnellen Fortschritts in der Computertechnologie sollte der Server so gewählt werden, dass er einfach durch ein stärkeres Modell ersetzt werden kann.

Computerspezifische Angaben zur heute notwendigen Arbeitsspeicher- und Prozessorleistung einer Praxisorganisation können an dieser Stelle kaum geliefert werden, da entsprechende Angaben bereits in 1-2 Monaten wieder überholt sind. Allerdings lohnt sich immer eine Leistungsanpassung oder -steigerung der Rechner an die speziellen technischen Anforderungen und Spezifikationen der digitalen Praxisgeräte wie z. B. Röntgengerät, Stereofotografie und Intraoralscanner. In diesem Zusammenhang sollte man sich be- 
wusst sein, dass die Leistung dieser Geräte häufig unabhängig von der verbauten Hardware (z. B. Optik) sind, und nur mit einem Software-Update der jeweiligen Firmen gesteigert werden können, also sollte der Server im Hintergrund bereits auf eine gewisse Leistungssteigerung vorbereitet sein.

Ganz wichtig und absolut unerlässlich ist die tägliche Datensicherung der Praxisdaten einschließlich Datenschutz. Damit ist kein brandsicherer Archivschrank gemeint, sondern die Notwendigkeit einer täglichen fragmentierten (die neu hinzugekommene Datenmenge eines Arbeitstages) Sicherung und einmal wöchentlich einer übergeordneten kompletten Datensicherung der Praxisdaten. Dies geschieht am besten an mehreren Orten. Lokal in der Praxis mit einem Netzwerkspeicher (NAS - Net-Attached-Storage), zu Hause und eventuell noch extern bei einem Datensicherungsunternehmen. Natürlich besteht auch die Möglichkeit, die tägliche Sicherung mittels externer Festplatte (früher Magnetbänder) durch einen Mitarbeiter/eine Mitarbeiterin von der Praxis aus durchführen zu lassen. Jedoch sollte man hierbei bedenken, dass jeder automatisierte Schritt, unabhängig von wechselndem Personal grundsätzlich sicherer ist.

\section{Grenzen und mögliche Stolpersteine digitaler Anwendungen}

Die Grenzen beginnen mit der persönlichen Haltung gegenüber Neuerungen im beruflichen Alltag. Wann immer die nächste noch bessere Version einer Software, einer Kamera oder eines Röntgengerätes abgewartet wird, besteht die Gefahr, dass eine Modernisierung komplett verpasst wird. Dies paart sich häufig mit der Angst vor den finanziellen Auslagen und dem Gedanken oder der Befürchtung, dass sich eine Investition in neue Technologien nicht mehr lohnen könnte.

Dass die hohen Anschaffungskosten und Lizenzgebühren bei verschiedenen Herstellern teilweise abschreckend sind, ist verständlich, dennoch gibt es häufig Möglichkeiten, sich mit Dentallaboren, Fräs- und Druck-Zentren zu arrangieren und die bestmögliche und preiseffizienteste Ausrüstung zu beschaffen.

Sobald die Hürde genommen ist und die Praxis mit neuen Geräten ausgerüstet wurde, ist es extrem wichtig diese auch zu benutzen, da bei fast allen digitalen Hilfsmitteln ein große Lernkurve zu erwarten ist. Frustration bis zum Erfolg und flüssigen täglichen Gebrauch erfordert manchmal 1-2 Monate.

Ein weiteres aktuelles Hindernis sind die noch fehlenden Materialien, womit mundbeständige FDA-geprüfte und ISO-genormte druck- oder fräsbare Produkte für die unterschiedlichen Anforderungen in der Kieferorthopädie gemeint sind. Die Industrie arbeitet mit Hochdruck daran diese zur Verfügung zu stellen (z. B. Nextdent, 3D-Systems), um auch in der Kieferorthopädie einen ähnlich effizienten Workflow zu gewährleisten wie in der rekonstruktiven Zahnmedizin.

Eine weitere Grenze ist das Vorstellungsvermögen bestehende Apparaturen zu modifizieren und in die digitale Welt zu transferieren oder gegebenenfalls neu zu entwickeln. Der Wille zur Pionierarbeit ist nicht jedermanns Sache. Aber auch hier holt die Industrie auf, und stellt viele Behandlungsmöglichkeiten anhand von digita- len Abformungen zur Verfügung (z. B. Invisalign, Align Technologies, San Jose, CA, USA).

\section{Gedruckte und metallgedruckte Appara- turen - Fiktion oder Wirklichkeit?}

Al Mortadi [15-17] war einer der Pioniere, der 2015 [15] eine gedruckte kieferorthopädische Apparatur vorstellte, bei welcher der Acryl-Anteil und die Halteelemente aus Metall gedruckt und zu einer aktiven Platte zusammengefügt wurden. Diese Apparatur wurde jedoch nur hergestellt, um die Machbarkeit eines neuen Herstellungsverfahrens zu prüfen. Allerdings war die Apparatur wegen der fehlenden federelastischen Eigenschaften der Metalllegierung (Remaniumstar, Dentaurum, Ispringen, Deutschland) damals noch nicht alltagstauglich.

Bis heute werden herausnehmbare Apparaturen nach wie vor auf 3D-gedruckten Modellen in konventionell labortechnischen Laborverfahren hergestellt. Der Aufwand und der Ertrag bei kombinierten gefrästen/gedruckten Acryl-Basen, bei denen aktive Elemente anschließend auf einem Modell zusammengefügt werden, sind im Moment noch zu groß und zu kostenintensiv.

Im Vergleich dazu werden aber bereits heute schon okklusale Splints [18] für Umstellungsosteotomien oder Knirscherschienen aus soliden Acrylblöcken herausgefräst oder durch 3-dimensionale Verfahren gedruckt (3D-Druck).

Generell kann man feststellen, dass alle Apparaturen, die keine zusätzlichen Elemente (Metallklammern oder Schrauben) enthalten und lediglich eine Kunststoffbasis benötigen, zunehmend bereits heute schon, bei entsprechender technischer Voraussetzung, hergestellt werden können.

Graf zeigt 2017 [19] anhand einer forcierten Dehnapparatur ( $\triangleright$ Abb. 1 und $\triangleright$ Abb. 2) die Möglichkeit, kieferorthopädische Apparaturen aus Metall mittels CAD/CAM (computer aided design/ computer aided manufacturing)-Verfahren umzusetzen. Dieses Verfahren, das Graf seit 2014 anwendet, beruht auf einer Software (Appliance Designer, 3Shape, Kopenhagen, Denmark), die den Import von STL (3D-Datensätze)-Files und das Designen von eigenen 3D-Stukturen und das Kombinieren mit weiteren Datensätzen erlaubt. So wird die Basis der forcierten Dehnapparatur an die Zähne des virtuellen Abdruckes designt und die Dehnschraube aus einer digitalen Bibliothek eingefügt. Die so entstandene Struktur wird nun additiv im selektiven Lasermelting aus Remaniumstar hergestellt ( $\triangleright$ Abb. 1 und $\triangleright$ Abb. 2 ).

Natürlich wäre das Wachs-Drucken eines Gießkörpers mit anschließendem klassischen Gussverfahren oder Fräsen auch möglich, aber wenn man den Verlauf der Entwicklung im Rapid-Prototyping beobachtet, ist der additive Fertigungsweg der Weg der Zukunft.

Abschließend wird die Dehnschraube mittels Laserschweißen in die Basis eingefügt. Und fertig ist die Apparatur. Ohne unnötige physische Modelle, was natürlich die Kosten und Umweltbelastung reduziert.

Auch die Herstellung von weiteren kieferorthopädische Apparaturen aus Metall wie z. B. Transpalatinal- und Lingualbögen ( $\triangleright$ Abb. 3) oder Herbstscharniere haben Graf und sein Team bereits erfolgreich in die digitale Welt übertragen. 

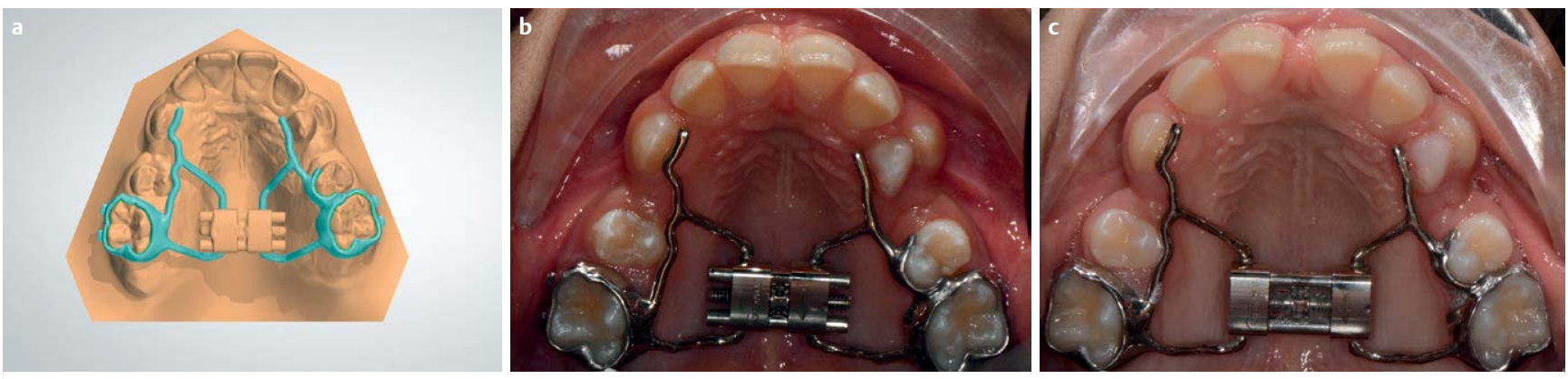

- Abb. 1 a Computergestütztes Design und Herstellungsverfahren einer Hyrax-Apparatur zur transversalen Dehnung des Oberkiefers; b die Apparatur nach Applikation und $\mathbf{c}$ nach transversaler Dehnung intraoral.
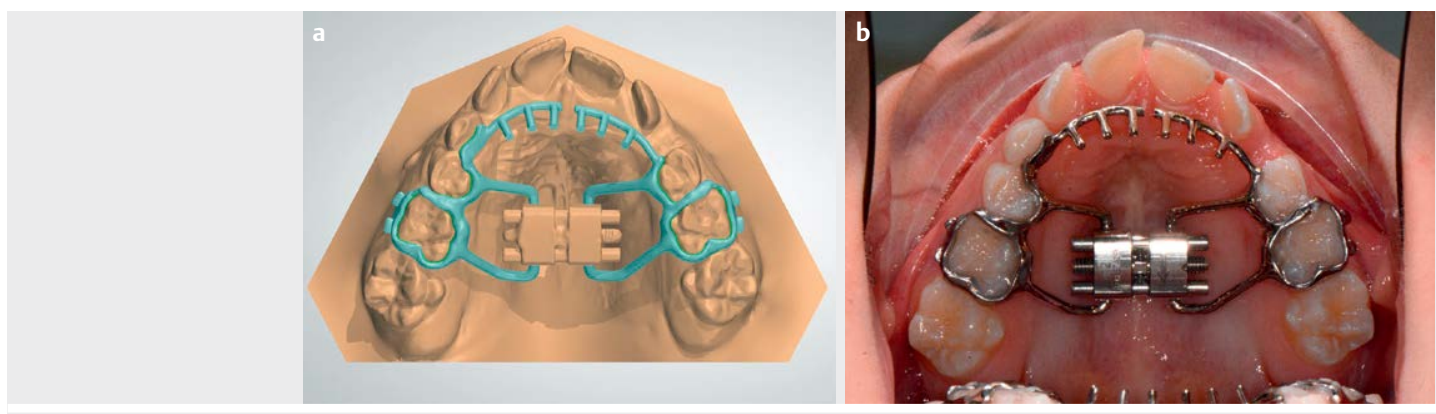

- Abb. 2 a Computergestütztes Design einer Hyrax-Apparatur in Kombination mit einem Zungengitter und b die klinische Situation nach intraoraler Applikation.
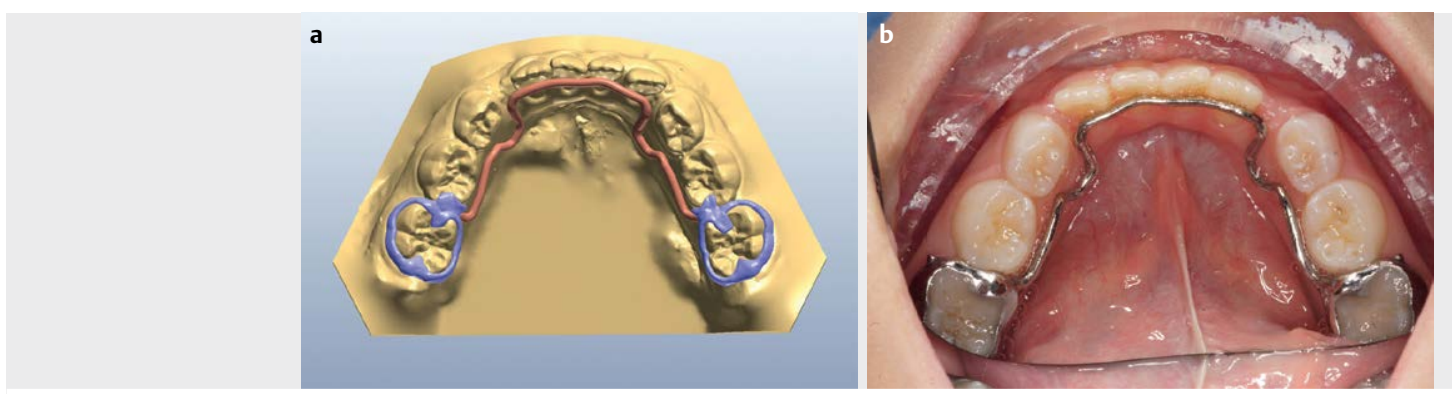

Abb. 3 a Computergestütztes Design eines Lingualbogens und b die klinische Situation nach intraoraler Applikation des Bogens.

Der große Vorteil bei diesen Apparaturen ist, dass im Falle eines Defektes die STL-Files des entsprechenden Patienten wieder verwendet werden können und jederzeit abrufbar sind. Die Apparatur kann damit an der Stelle des Defektes digital nachgebessert und jederzeit wieder ausgedruckt werden. Ohne zusätzliche Termine, zusätzliche Abformungen oder weitere Belastungen für den $\mathrm{Pa}$ tienten.

Skelettal gestützte Apparaturen wie das Benefit-System (B. Wilmes, Universität Düsseldorf) ( $\triangleright$ Abb. 4) können ebenfalls adaptiert werden. Das Interessante an dem 3Shape-Scanner ist, dass die Implantate ohne zusätzliche Abformungspfosten eingescannt werden können. Das gescannte Implantat wird im digitalen Abdruck durch ein digitales Implantat-Analog ersetzt, und die Basisstruktur der Apparatur anschließend direkt konzipiert.
Neben diesen Apparaturen eröffnen sich noch viele weitere Möglichkeiten: das virtuelle Setup anhand eines Tastenklicks, um dem Patienten zu zeigen was möglich ist.

Indirektes Kleben, respektive das Positionieren der Brackets, kann am Computerdisplay erledigt werden und danach wird das Transfer-Tray direkt ausgedruckt. In dieses können anschließend die Brackets nur noch eingeklickt, mit Adhäsiv versehen und im Patientenmund eingesetzt werden.

Auch die Schienentherapie wird revolutioniert werden, sobald ähnliche Materialien direkt als Schienen gedruckt werden können, und nicht mehr über Gips- oder gedruckte Modelle tiefgezogen werden müssen.

Die geklebten Retainer können schon länger entweder anhand eines ausgedruckten Scans von Eckzahn bis Eckzahn (keine kom- 


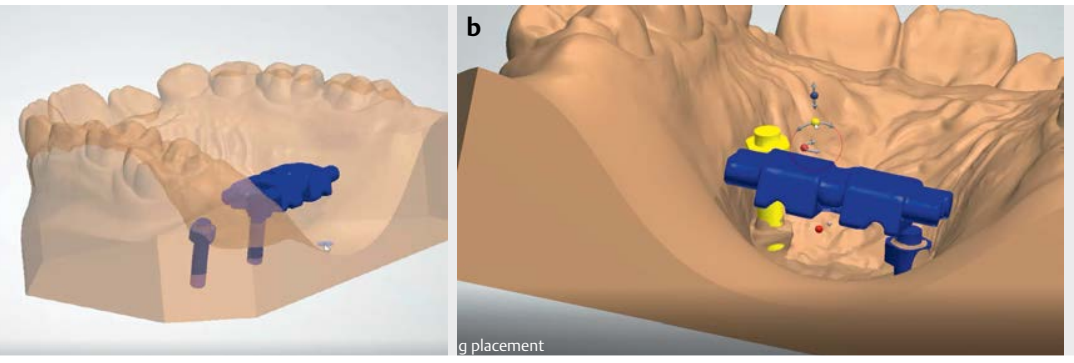

- Abb. 4 a-b Computergestützte Design einer skelettal gestützten Apparatur zur transversalen Dehnung des Oberkiefers.

plette Kieferabformung notwendig!) herkömmlich hergestellt werden. Dann gibt es noch die Möglichkeit, diese in 3D-gefräst herstellen zu lassen, oder in 2D nur auf einer Ebene mit Laser aus einer NiTi-Platte herausschneiden zu lassen (20; Memotain CA Digital, Mettmann, Deutschland).

Zusammenfassend lässt sich also sagen, dass gedruckte und metallgedruckte Apparaturen heute schon Realität geworden und weit weg von Fiktion sind. Das wird und ist die Zukunft.

\section{Schlussbetrachtung}

Wir leben in einer extrem spannenden Zeit mit unglaublich raschen Entwicklungen, aber auch unglaublichen Möglichkeiten im Hinblick auf die eigene Kreativität. Selten war es mit so wenig Aufwand möglich, so viele unterschiedliche Aspekte einer einfachen kieferorthopädischen Apparatur mit nur einem Tastenklick zu verändern und individuell anzupassen. Diese Entwicklung wird noch eine Weile anhalten, bis es für jeden mittels weniger Klicks einfach möglich ist, seine gewünschte Apparatur von einer Software designen und herstellen zu lassen.

Jeder muss sich selber fragen, inwieweit er in seiner Praxis bei dem digitalen „Wettrüsten“ mitmacht. Ob es sich lohnt für eine Praxis alle aufwändigen Investitionen zu tätigen, spezialisierte Informatiker einzustellen, oder ob es sinnvoller ist diese Arbeiten an ein spezialisiertes Zentrum weiterzugeben. Diese Zentren können natürlich durch mehrere Kieferorthopäden gemeinsam gestaltet werden.

Man sollte auch die Überlegung anstellen, ob man sich in der durch das Digitalisieren frei gewordenen Zeit eher neuen Patienten widmen will, anstatt viele Stunden an einem Rechner zu verbringen, um Probleme zu lösen, welche eine spezialisierte Fachkraft viel effizienter lösen kann. Das kommt immer auf die Einstellung und Motivation des Einzelnen an, und darauf, wo er sich positionieren möchte: eher am Patienten oder im Büro vor dem Computer.

\section{Literatur}

[1] Dawood A, Marti Marti B, Sauret-Jackson V et al. 3D printing in dentistry. Br Dent J. 2015; 219: 521-529 doi:10.1038/sj.bdj.2015.914

[2] Wesemann C, Muallah J, Mah J et al. Accuracy and efficiency of full-arch digitalization and 3D printing: A comparison between desktop model scanners, an intraoral scanner, a CBCT model scan, and stereolithographic 3D printing. Quintessence Int 2017; 48: 41-50 doi:10.3290/j.qi.a37130
[3] Aragón ML, Pontes LF, Bichara LM et al. Validity and reliability of intraoral scanners compared to conventional gypsum models measurements: a systematic review. Eur J Orthod 2016; 38: 429-434 doi:10.1093/ejo/cjw033

[4] Brown MW, Koroluk L, Ko CC et al. Effectiveness and efficiency of a CAD/CAM orthodontic bracket system. Am J Orthod Dentofacial Orthop. 2015; 148: 1067-1074 doi:10.1016/j.ajodo.2015.07.029

[5] Müller-Hartwich R, Präger TM, Jost-Brinkmann PG. SureSmile - CAD/ CAM system for orthodontic treatment planning, simulation and fabrication of customized archwires. Int J Comput Dent 2007; 10: 53-62

[6] Pauls A, Nienkemper M, Schwestka-Polly R et al. Therapeutic accuracy of the completely customized lingual appliance WIN: A retrospective cohort study. J Orofac Orthop 2017; 78: 52-61 doi:10.1007| s00056-016-0058-9

[7] Schulz R. Digitale Volumentomografie - Möglichkeiten und Grenzen in der regulären (primär nicht-chirurgischen) kieferorthopädischen Diagnostik und Therapie. Inf Orthod Kieferorthop 2016; 49: 145-150

[8] Schulze R, Deppe H, Betz W et al. s2k-Leitlinie „Dentale digitale Volumentomographie“. 2013; Available from: URL http://www.awmf. org/leitlinien/detail/II/083-005.html

[9] European Commission. Radiation protection No 172: Cone Beam CT for Dental and Maxillofacial Radiology. Evidence Based Guidelines: A report prepared by the Sedentexct Project 2012 (v2.0)

[10] Solaberrieta E, Garmendia A, Brizuela A et al. Intraoral Digital Impressions for Virtual Occlusal Records: Section Quantity and Dimensions. Biomed Res Int 2016; 2016: 7173824 doi:10.1155/2016/7173824

[11] Patzelt SBM, Lamprinos C, Stampf S et al. The time efficiency of intraoral scanners: an in-vitro comparative study. Journal of the American Dental Association 2014; 145: 542-551

[12] Patzelt SBM, Emmanouilidi A, Stampf S et al. Accuracy of full-arch scans using intraoral scanners. Clinical Oral Investigations. 2014; 18: 1687-1694 doi:10.1007/s00784-013-1132-y

[13] Zhang F, Suh KJ, Lee KM. Validity of Intraoral Scans Compared with Plaster Models: An In-Vivo Comparison of Dental Measurements and 3D Surface Analysis. PLoS One 2016; 11: e0157713 doi:10.1371/ journal.pone.0157713 eCollection 2016

[14] Camardella LT, Breuning H, de Vasconcellos Vilella O. Accuracy and reproducibility of measurements on plaster models and digital models created using an intraoral scanner. J Orofac Orthop 2017; 78 (3): 211-220

[15] Al Mortadi N, Jones Q, Eggbeer D et al. Fabrication of a resin appliance with alloy components using digital technology without an analog impression. Am J Orthod Dentofacial Orthop. 2015; 148: 862-867 doi:10.1016/j.ajodo.2015.06.014

[16] Al Mortadi N, Eggbeer D, Lewis J et al. CAD/CAM/AM aplications in the manufacture of dental appliances. Am J Orth Dentofacial Orthop 2012; 142: 727-733 
[17] Al Mortadi N, Lewis J, Eggbeer D et al. Design and fabri tion of a sleep apnea device using computer-aided design/addit manufacture technologies. Proc Inst Mech Eng H 2013; 227: 350

[18] Goiato MC, Santos MR, Pesqueira AA et al. Prototyping for surgical and prosthetic treatment. J Craniofac Surg 2011: 22; 914-917
[19] Graf S. Computer-aided design and manufacture of hyrax devices: Can we go digital... for real? AJODO in press 2017

[20] Kravitz ND, Grauer D, Schumacher P et al. Memotain: A CAD/CAM nickel-titanium lingual retainer. Am J Orthod Dentofacial Orthop 2017; 151: 812-815 